\title{
CHEMICAL EVOLUTION IN SPACE - A SOURCE OF PREBIOTIC MOLECULES
}

\author{
J. Mayo Greenberg \\ Laboratory Astrophysics, University of Leiden, P.O. Box 9504, \\ 2300 RA Leiden, The Netherlands
}

\section{ABSTRACT}

In Laboratory Astrophysics at Leiden Unfversity a laboratory analog for following the chemlcal evolution of interstellar dust in space shows that the dust contains the bulk of organic material in the universe. We follow the photoprocessing of low temperature (10 $\mathrm{K}$ ) inixtures of lces subjected to vacuum ultraviolet radiation in simulation of interstellar conditions. The most important, but necessary, difference is in the time scales for photoprocessing. One hour in the laboratory is equivalent to one thousand years in low density regions of space and as much as, or greater than, ten thousand to one milion years in the depths of dense molecular clouds. The ultimate product of photoprocessing of grain material in the laboratory is a complex nonvolatile residue which is yellow in color and soluble in weter and methenol. The molecular weight 16 greater than the mid-hundreds. The 1nfrared absorption spectre indicate the presence of carboxylic acid and antno groups resembling those of other molecules of presumably preblological significance produced by more classical wethods. One of our resfdues, when subjected to high resolution mass spectroscopy gave mask of 82 corresponding to $\mathrm{C}_{4} \mathrm{H}_{6} \mathrm{H}_{2}$ after release of $\mathrm{CO}_{2}$ and trace ammounts of urea auggesting anino pyroline rings.

The deposit of preblotic dust molecules occurred as many as 5 tines in the first $500-700$ mililon years on a primitive Earth by accretion during the pasege of the solar system through a denge interstellar cloud. The deposition rate during esch pasage is.estimated to be between $10^{9}$ and $10^{10} \mathrm{~g}$ per year during the million or so years of each passage; $1 . e$. a total deposition of $10^{9}-10^{10}$ metric tons of complex organic material per passage.

\section{INTRODUCTION}

Although the jor obvious constituents of our Milky Way are the stars, the space between the stars 18 far from empty. Indeed it 18 from the matter wich fills this opace that new stars are continually being born. What is this interstellar waterial and whet role can it possibly play in the story of the origin of life?

Atons. Most of the interstellar medium is hydrogen wich was created in the earliest stages of our universe. The formation of the heavier elements has been an ongoing process ever since the first gtars were born. What w see todey, on the grand ecale, is distribution of the elements which have been produced by stars and ejected back into epace. Following helium which plays no role in the interstellar chemistry, the group of elements comprising oxygen, carbon and nitrogen constitute about one part in a thounand by number relative to hydrogen. The next most abundant group magnesium, sil1con, 1ron and sulphur- are less abundant by a factor of ten: It 18 obvious that in space the elements which are required for organic molecules are the most abundant.

Molecules and Dust. Since the advent of the discovery of formaldehyde (H,CO) in the interstellar gas in 1969 [1] a wide range of molecular epecles have been detected. The most abundant of these is carbon monoxide (CO) and a 11 sting of the molecules detected to date (see Table 1) is convincing evidence that there is a very active chemical factory in space [2]. However, as we shall see, these are only the "tip of the iceberg". The most complex and abundant molecules are not in the gas but rather must be in smail solid particles called interstellar dust wich float about in the gas. What these dust particles are, both chemically and physically, will therefore be our principal theme. However, flrst let us consider where and how much dust there is, and how this relates to the amount and presence of hydrogen and other molecules. 
TABLE 1. Molecules and Ions Identified in the Interstellar Gas.

$\begin{array}{llllll}\mathrm{CN} & \mathrm{NS} & \mathrm{SO}_{2} & \mathrm{C}_{3} \mathrm{~N} & - & \mathrm{HC}_{5} \mathrm{~N} \\ \mathrm{CH} & \mathrm{C}_{2} & \mathrm{C}_{2} \mathrm{H} & \mathrm{HNCS} & \mathrm{CH}_{3} \mathrm{CN} & - \\ \mathrm{CH}^{+} & \mathrm{NO} & \mathrm{N}_{2} \mathrm{H}^{+} & \mathrm{C}_{2} \mathrm{H}_{2} & \left(\mathrm{NH}_{2}\right) \mathrm{HCO} & \mathrm{CH}_{3} \mathrm{COOH} \\ \mathrm{H}_{2} & - & \mathrm{HCO} & - & \mathrm{CH}_{3} \mathrm{OH} & - \\ \mathrm{OH} & \mathrm{H}_{2} \mathrm{O} & \mathrm{HCS}^{+} & \mathrm{HC}_{3} \mathrm{~N} & \mathrm{CH}_{3} \mathrm{SH} & \mathrm{C}_{2} \mathrm{H}_{5} \mathrm{OH} \\ \mathrm{CO} & \mathrm{HCN} & - & \mathrm{HCOOH} & - & \left(\mathrm{CH}_{3}\right)_{2} \mathrm{O} \\ \mathrm{CS} & \mathrm{HCO}^{+} & \mathrm{NH}_{3} & \mathrm{H}_{2} \mathrm{CNH} & \mathrm{CH}_{3} \mathrm{COH} & \mathrm{CH}_{3} \mathrm{CH}_{2} \mathrm{CN} \\ \text { S1O } & \mathrm{HNC} & \mathrm{H}_{2} \mathrm{CO} & \mathrm{H}_{2} \mathrm{NCN} & \mathrm{CH}_{3} \mathrm{NH}_{2} & \mathrm{HC}_{7} \mathrm{~N} \\ \text { SO } & \mathrm{OCS} & \mathrm{H}_{2} \mathrm{CS} & \mathrm{H}_{2} \mathrm{C}_{2} \mathrm{O} & \mathrm{CH}_{3} \mathrm{CHCN} & - \\ \text { S1S } & \mathrm{H}_{2} \mathrm{O} & \mathrm{HNCO} & \mathrm{HC}_{4} & \mathrm{CH}_{3} \mathrm{C}_{2} \mathrm{H} & \mathrm{HC}_{9} \mathrm{~N}\end{array}$

Distribution of Gas and Dust. In Figure 1 we see an example of a spiral galaxy simflar to ours (MS1). The spiral arms are seen because they contain the brightest stars. However, the spiral arms are perhaps even better delineated by the dark lanes at the Inner edges of the arms. These dark lanes are produced by the blocking of the 11 ght of the stars by concentrat1ons of the Interstellar dust acting Just like a smoke screen. In F1g. I s superposit1on of the peak gas contours is seen to closely colnclde with the dark dust lanes. This show that where the dust is dense so Is the gas; 1.e. the dust 18 correlated with the gas. F1g. 2 shows how a spiral galaxy looks when seen edge-on. The dark band separating the two halves of the galaxy is again produced by the light blocking power of the dust whlch is concentrated in a thin disk within the galaxy of stars. It turns out, from observat1ons of the gas, that the $g^{28} 18$ also concentrated within a thin disk along with the youngest population of stars.

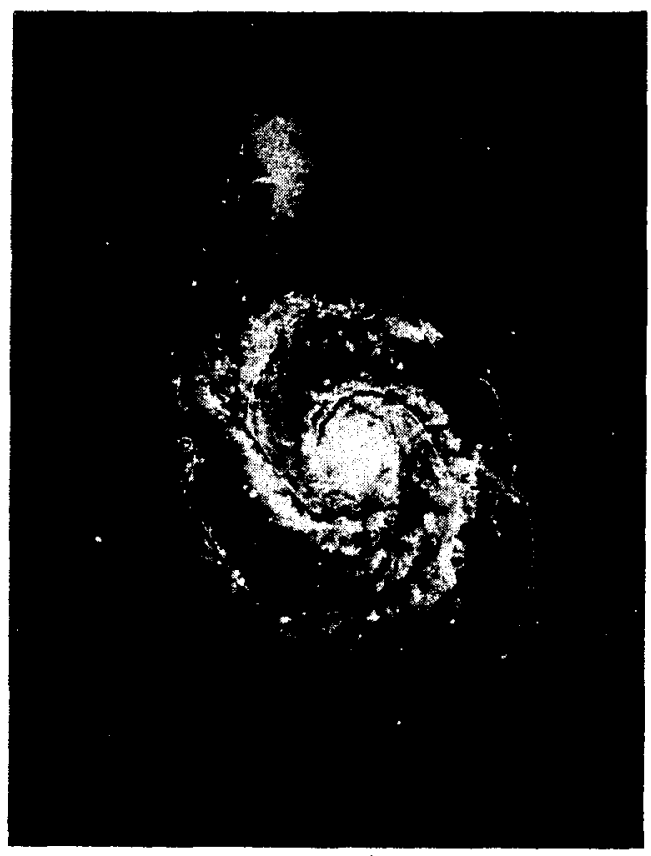

F1g. 1. Photograph of a spiral galaxy (MS1) seen face-on. The dark bands at the inner edges of the spiral arms are the clearly defined concentrations of the dust. Superimposed on the photograph are solid lines showing the peak in the continuum radiation at $1415 \mathrm{MHz}$. These lines appear to coincide well with the dust lanes (From Mathewson et al. 1972, Astron. Astrophys. 17, 468). 


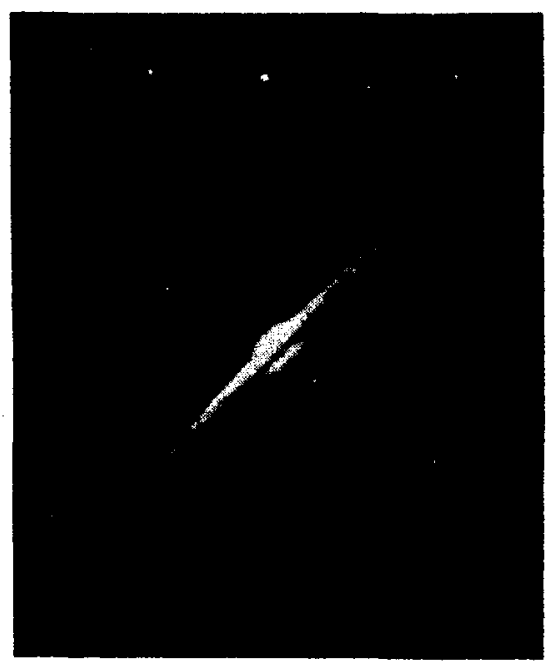

Fig. 2. The distant spiral galaxy NGC 4565. This galaxy seen on edge exhibits a dark band in its central plane, indicating the concentration of extinction by Interstellar dust.

Although we can say that the dust and gas have a well-defined general distribution within the galaxy, a brief glance at the night sky reveals a highly inhomogeneous and patchy structure. The interstellar medium actually consists of a chaotic distribution of gas/dust clouds with a varlety of densities always in motion and passing from one phase to another in their evolution with the most dramat1c phase appearing when new stars are formed. The widest variety of molecules is associated with clouds in intimate association with star formation. These clouds usually have densities of hydrogen greater than $n_{H}=100 \mathrm{~cm}^{-3}$ and are called dense or molecular clouds.

From the combination of observations of the mean amount of starlight extinction per unit distance and from the mean size of the dust grains which we infer from the color dependence of this extinction we can derive a mean spatial number density of about one particle per $10^{12} \mathrm{~cm}^{3} ;$ 1.e. about one particle in a cube whose sides are 100 meters- larger than football fleld!' In dense clouds this density may be higher by a factor of $10^{4}$ to $10^{6}$ and, in protostellar nebulae, much higher, Golng with the dust, or vice verse, is a mean hydrogen density of about $n_{H}=1 \mathrm{~cm}^{-3}$. We see that, by terrestrial standards, even in dense interstellar cloud the pressure is $\mathrm{p} \approx 3 \mathrm{n}_{\mathrm{y}} 10^{-20} \mathrm{mbar}$ which is a very good vacuum. However the vastness of space more than compensates for the low density when one considers the total mass of the 1nterstellar material. For example, although the one hydrogen atom per cubic centimeter gives an average spatial density of $1.66 \times 10^{-24} \mathrm{~g} \mathrm{~cm}^{-3}$, the mean stellar number density gives only a factor of ten greater; 1.e. the mass of gas (mostly hydrogen) relative to stars is about $10 \%$. Simllarly the mas density of the dust relative to gas turns out to be about $1 \%$, so that relative to the stars it $181+1000$. Th1s 18 large amount when compared with estimates of the total planetary mass. In our solar system, for example, the mass of all planets together-mostly in Jupiter-amounts to about $1+1000$ relative to the sun's mess. Therefore, even if every star in our Milky Way has a planetary system like ours, their mass would not exceed that of the interstellar dust. One can therefore state with confidence that the overall mass of the small dust particles in space probably exceeds that of all possible Earth-like planets by many orders of magnitude. We shall come back to this later because a mere comparison of relative masses of two species of objects does not necessarily define their relative importance in all contexts.

The molecules are generally concentrated in the denser regions in space although evidence for some molecules $11 \mathrm{ke} \mathrm{HC}_{5} \mathrm{~N}, \mathrm{H}_{2} \mathrm{CO}$ and certainly $\mathrm{CO}$ is seen in relatively tenuous regions called diffuse clouds with hydrogen densities of $\mathrm{n}_{\mathrm{H}} \approx 10 \mathrm{~cm}^{-3}$. Although the total number of molecules identified is large, about 50 , their absolute number is, with the exception of $\mathrm{CO}$, quite mall with respect to the cosmic abundance availability of their constituents. In fact, if we count all the molecules seen and compare their total mass with that of the dust it is less by a factor of at least 100 unless we include $C O$ in which case it may be as large as, but generally considerably less than, $1 / 2$. 
The Interstellar Environment. The typical gas kinetic temperatures of diffuse clouds is $T=$ $100 \mathrm{~K}$. For molecular clouds the temperaţure range is $10 \mathrm{~K} \leqq \mathrm{~T} \leqq 50 \mathrm{~K}$. Thus in a diffuse cloud with a mean density $\mathrm{n}_{\mathrm{y}} \simeq 10 \mathrm{~cm}^{-3}$ a hydrogen atom has $\tilde{a}$ typlcal speed of about $v_{H}=1.5 \mathrm{~km} \mathrm{~s}^{-1}$ and in a molecular cloud, with mean density $\mathrm{n}_{\mathrm{H}}>1000 \mathrm{~cm}^{-3}, \mathrm{~T} \approx 10-50 \mathrm{~K}$ so that a CO molecule has a gas kinetic speed of about $\mathrm{v}_{\mathrm{CO}}=0.1 \mathrm{~km} \mathrm{~s}^{-1}$.

In Table 2 the mean propertles of the fnterstellar medium are summarized.

TABLE 2. Average Propertles of Gas, Radiation and Dust in the Interstellar Medium

$\underline{\text { Gas }}$

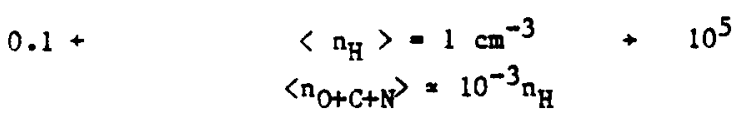

$$
\begin{aligned}
& 0: C: N \approx 6.8: 3.7: 1 \\
& \left\langle\mathrm{n}_{\mathrm{Mg}+\mathrm{S} 1+\mathrm{Fe}}\right\rangle=10^{-4} \mathrm{n}_{\mathrm{H}} \\
& \mathrm{Mg}: \mathrm{S} 1: \mathrm{Fe}=1: 1: 1 \\
& 10,000 \mathrm{~K}+\quad \mathrm{T}_{\mathrm{gas}}=100 \mathrm{~K} \quad+10 \mathrm{~K}
\end{aligned}
$$

\section{U1traviolet Radiation in Low Density Clouds}

$$
\begin{gathered}
\left\langle\mathrm{n}_{\lambda\langle\lambda}\right\rangle=3 \times 10^{-3} \mathrm{~cm}^{-3} \\
\lambda_{t}=2000 \AA: \mathrm{hv}_{\mathrm{t}}=6 \mathrm{eV} \\
\frac{\text { Dust in Low Dens1ty Clouds }}{\text { Core mantle part1cles }(\equiv \mathrm{c}-\mathrm{m})} \\
\text { a core }=0.05 \mu \mathrm{m}, \overline{\mathrm{a}} \text { mantle }=0.12 \mu \mathrm{m} \\
\left\langle\mathrm{n}_{\mathrm{d}}\right\rangle=10^{-12} \mathrm{n}_{\mathrm{h}} \equiv\left\langle\mathrm{n}_{\mathrm{c}-\mathrm{m}}\right\rangle \\
\mathrm{T}_{\text {dust }}=10 \mathrm{~K}
\end{gathered}
$$

Very Small Bare (b) Particles

$$
\begin{aligned}
& a_{b}=0.005 \mu \mathrm{m} \\
& \left\langle n_{b}\right\rangle=10^{3}\left\langle n_{d}\right\rangle
\end{aligned}
$$

Close to hot young stars the temperature of the gas can be as $\mathrm{high}$ as $10,000 \mathrm{~K}$ in a region of fully lonlzed hydrogen thus an HII (or lonlzed hydrogen) reglon. In the cool clouds, the hydrogen is un-ionized. We refer to these as HI regions.

The megn radiation energy density coming from the general stellar population is about 0.5 $\mathrm{eV} \mathrm{cm}^{-3}$ of which about $0.003 \mathrm{eV} \mathrm{cm}^{-3}$ is in ultraviolet photons with energy greater than 6 ev. This energy is selected as a rough threshold value for photodissoriation of most molecules (see Table 3).

The Interstellar medium is far from thermal equilibrium. Curlously, although both the gas temperature and the dust temperature are basically governed by the radiation from stars, the dust is generally much colder than the gas, the only exceptions being in reglons of very high gas density. The mean temperature of the dust grains, which is reached by a balance between absorption of radiation from the amblent fleld and emission of radiation by the small dust gra1ns, $1 \mathrm{~s}$ only about $10 \mathrm{~K}$ [3]. It is only near energetfc sources of radiation that grain temperatures get as high as 50 or $100 \mathrm{~K}$. of course, very close to hot stars temperatures in the thousands, leading to complete evaporation, occur. But this affects only a very small fraction of all the dust grains at any one time. 
TABLE 3. Some Approximate Molecule and Radical Bond Dissoclation Energies Adopted from Calvert and P1tts, 1966, Photochemistry, J. Wiley, and Sons, N.Y.

\begin{tabular}{|c|c|c|c|c|c|}
\hline Bond & d Broken & $\Delta \mathrm{E}$ & Bond & Broken & $\Delta \mathrm{E}$ \\
\hline & $\mathrm{R}^{\mathrm{R}} \mathrm{R}^{\prime}$ & $(\mathrm{ev})$ & $\mathrm{R}-\mathrm{F}$ & & $(e V)$ \\
\hline $\mathrm{OH}$ & $-\quad \mathbf{H}$ & 5.14 & $\mathrm{CH}_{2} \mathrm{CH}$ & $-\mathrm{H}$ & 4.54 \\
\hline 0 & $-\quad H$ & 4.40 & $\mathrm{CH}_{3}$ & $-\mathrm{CH}_{2} \mathrm{O}$ & 0.54 \\
\hline 0 & -0 & 5.16 & $\mathrm{OCH}_{2}$ & $-\mathrm{H}$ & 1.02 \\
\hline $\mathbf{S}$ & -0 & 5.44 & $\mathrm{HCO}$ & $-\mathrm{H}$ & 3.79 \\
\hline os & -0 & 5.66 & $\mathrm{H}_{2} \mathrm{C}$ & -0 & 7.59 \\
\hline $\mathbf{H}$ & $-\quad H$ & 4.50 & $\mathrm{CH}_{3} \mathrm{CO}$ & $-\mathrm{H}$ & 3.79 \\
\hline$C$ & -0 & 11.17 & $\mathbf{H}$ & $-c 0$ & 0.755 \\
\hline OC & -0 & 5.48 & $\mathrm{C}_{2} \mathrm{H}_{5}$ & $-\mathrm{H}$ & 4.27 \\
\hline $\mathrm{CH}_{2}$ & $-\infty 0$ & 2.32 & $\mathrm{CH}_{3}$ & $-\mathrm{CH}_{3}$ & 3.62 \\
\hline $\mathrm{CH}_{3}$ & $-\mathrm{OH}$ & 3.84 & $\mathbf{N}$ & -0 & 6.52 \\
\hline $\mathrm{CH}_{3}$ & $-\mathrm{H}$ & 4.40 & $\mathrm{NH}_{2}$ & $-H$ & 4.47 \\
\hline$C$ & $-\quad H$ & 3.53 & $\mathrm{NH}$ & $-\mathrm{H}$ & 4.14 \\
\hline $\mathrm{CH}$ & $-\quad H$ & 5.48 & $\mathbf{N}$ & $-N$ & 9.78 \\
\hline
\end{tabular}

PHOTOCHEMICAL PROCESSING OF DUST.

Intersteller Grain Wodel. A working model for 1nterstellar grains [4] cons1sts primar1ly of two characteristic sizes of particles: 1) core-mantle particles of size $\% 0.12 \mu \mathrm{m}$ (radius) wth cores of sillcates of radius $\approx 0.05 \mu \mathrm{m}$ and mantles of accreted molecules made up mainly of oxygen, nitrogen, carbon and hydrogen; 2) Bare particles of size $\leq 0.01$ um probably made of individual particles of silicates, carbon and perhaps large organic molecules. The bulk of the mass of the dust is in the core-mantle particles and we shall limit our diecussion primarily to their chemical evolution.

While a core-mantle particle floats about in a cloud of gas it is continually being bomberded by energetic ultravlolet photons. These photons originate either in distant or nearby stars or arise from shocks produced by stellar winds within the clouds. If we were to consider the composition of a grain mantle as consisting, for example, of a frozen mixture of such oimple wolecules as water $\left(\mathrm{H}_{2} \mathrm{O}\right)$, wethane $\left(\mathrm{CH}_{4}\right)$ and amonia (NH3), as was proposed by Van de Hulst some 30 years ago [5] we real1ze that, in the course of some $t 1 \mathrm{me}$, depending on the ultraviolet flux, these molecules will be broken by photons which penetrate the grain. This is schematically 1llustrated in Figure 3 . It turns out that for a typical sized grain (semi thickness $a=0.12 \mu \mathrm{m}$ ) of such initial composition the flux $\phi_{\text {of }}$ ultraviolet photons in the mean interstellar medium is sufficlent to break every molecular bond in the grain in some tens to hundreds of years; hundreds if the flux in $\Phi=10^{8} \mathrm{~cm}^{-3} \mathrm{~s}^{-1}$ as given by Habing [6] or tens if as given by Metzger (private communication).

Since the time scales which prevall in the interstellar medium are generally in the range of $10^{6}-10^{8}$ years we see that a grain has litcle chance of remaining chenically static. What can occur within an individual grain as a result of ultraviolet photons is pictured in Figure 3. In the f1rst instance ultraviolet photons may break up the molecules which are in the grain, leaving the radical pieces frozen in. Sometimes these pleces may recombine to produce the original molecule: Sometimes, as plctured in the second step, adjacent radicals recombine to form new wolecules. And sometimes, radical may remain without reacting. At some stage, plctured in the last step of Figure 3 , we envisage a grain which consists of a reconstituted frozen melange of molecules and radicals of varying complexity. As we shall see, this sequence forms but one element in the overall chemical evolution of a grain mantle but $1 t$ is the basic process of photodissoclation which leads to all that follows.

Free Radical Storage, Trlggered Reactlons and Explosions. If a grain were to remaln at a constant temperature in a constant bath of ultraviolet radiation it would probably arrive at an ultimate steady state in time scales of $10^{6}$ - $10^{8}$ years so that the making and breaking of bonds and the mean molecular and radical composition would be on the average, unchanging. However, there are a number of sporadic events which may lead to a break in this state which, it turns out, is quite unstable. 


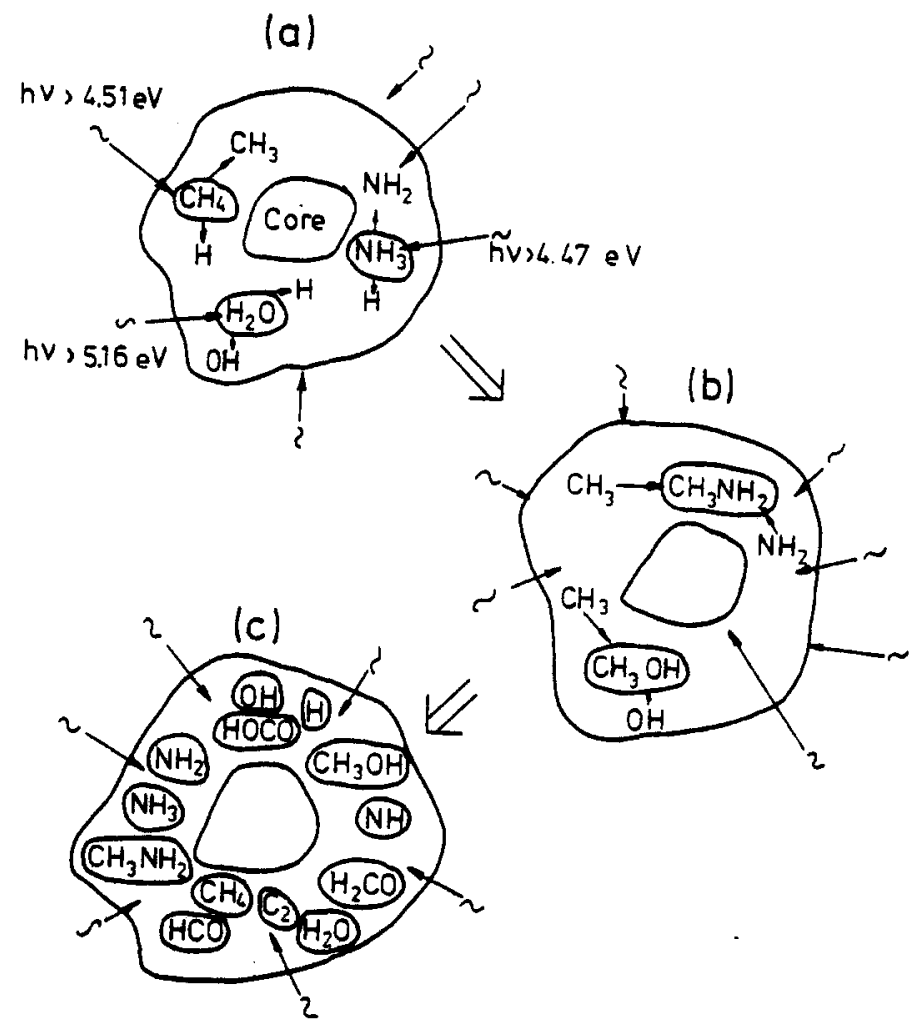

F1g. 3. Schematic evolution sequence for a grain mantle at $10 \mathrm{~K}$ subjected to ultraviolet photolys1s. The processes 1llustrated are photodissoclation, radicalradical combination, production of new molecules and radicals.

Free radicals are inherently highly reactive, containing an unpalred electron. When two free radicals come in contact they imediately combine, with no activation energy required, and release a significant amount of energy in the process, of the order, characteristically, of 4-5 eV. If enough free radicals (there is a critical number density) are stored in a grain, a triggering event may lead to a chaln reaction in which the heat generated by radical reections frees other frozen radicals and permits them to diffuse enough to find further radicals wth which to react, the sequence building up sometimes to an explosive reaction in the grain. It is only because the mean grain temperature is as Iow as $10 \mathrm{~K}$ that the radicals are normally prevented from readily diffusing to produce such chain reactions [7].

\section{LABORATORY ANALOG OF INTERSTELLAR GRAIN PHOTOCHEMTSTRY}

The Astrophysical Laboratory at the University of Lelden is the first to succeed in simulating the essential interstellar space conditions as they affect the evolution of Interstellar grains. Although the impetus of this work arose exclusively out of an attempt to answer purely astronomical questions, its extension to a possible connection with problems of preblological interest was quite natural, even inevitable.

In the 1940's the "d1rty 1ce" model was proposed for the interstellar dust. In th1s model atoms of oxygen, carbon, nitrogen and hydrogen were assumed to accrete on the particle surfaces and combline with hydrogen to form a frost of the saturated molecules water $\left(\mathrm{H}_{2} \mathrm{O}\right)$, methane $\left(\mathrm{CH}_{4}\right)$ and ammonia $\left(\mathrm{NH}_{3}\right)$ in relative abundances proportional to their cosmic abundances, thus leading to a particle dominated by $\mathrm{H}_{2} \mathrm{O}$ lce but including other ingredients; thus the name "dirty 1ce". 
With the introduction of new infrared detection techniques to astronomical problems it became possible in the mid-1960's to search for the expected strong $\mathrm{H}_{2} \mathrm{O}$ ice absorption bond at 3 microns due to the $0-H$ stretch [8]. The inftial results of such observations were negative in that they indicated $\mathrm{H}_{2} \mathrm{O}$ ice to be $f a r$ less abundant than predicted, this brought the dirty lce model into question. An alternative suggestion which allows for abundant oxygen in grains without much $\mathrm{H}_{2} \mathrm{O}$ is that the general vltraviolet radiation field in space is capable of breaking the $\mathrm{H}_{2} \mathrm{O}$ and other molecules in the solid particles so that in recombining, the oxygen need not return to the $\mathrm{H}_{2} \mathrm{O}$ form, and therefore need not exhibit the $3 \mathrm{um}$ feature. This proposal was given a strong additional push with the announcement of the discovery in space of the (then) surprisingly complex molecule formaldehyde ( $\mathrm{H}_{2} \mathrm{CO}$ ) by radio astronomers [9]. The obvious extension of the photodissociation-recombination proposal for reducing the $\mathrm{H}_{2} \mathrm{O}$ abundance was that a simple molecule $11 \mathrm{ke} \mathrm{H}_{2} \mathrm{CO}$ would be one of the most $11 \mathrm{kely}$ molecules produced by recombinations. The next step, to consideration of much more complex molecules, followed easily. Indeed, there was an analogy between the photochemical processing of the small interstellar grains [10] and the energetically induced (lightning, etc.) chemical processing in the presumptive primitive Earth's atmosphere as simulated by Miller and Urey [11].

However, though some of the processes which could be Induced by ultraviolet radiation in Interstellar grains must resemble those which were presumed to have occurred in the atmosphere, there are also some basic differences. One is that at the very low temperatures of grains the broken molecular species are inhlbited from moving freely to find a partner to recombine with. Secondly, the molecules in the solid are at all times in direct contact with each other rather than widely separated as in a gas, so that relaxation times are orders of magnitude smaller.

How the mixing and recombination occurs in the dust involves some processes, in addition to the basic ultraviolet photodissociation, which occur as the gralns move about in space.

Laboratory Methods. A schematic of the main elements of the experimental set-up is shown in Flgure 4. The two key components are the maintenance of low temperature and the ultraviolet radiation source. The low temperature is achleved by means of a closed cycle helium cryostat within which one gets temperatures as low as $10 \mathrm{~K}$ on "cold finger" which can varlously be an aluminfum block or a transparent window of glass, sapph1re or l1thium fluoride mounted on a metal ring. Varlous gases may be controllably allowed to enter the vacuum chamber of the cryostat (presaure down to $10^{-8}$ torr) via a capillary tube. These gases condense as a frost on the cold finger which acts then like the core of the 1nterstellar grains. On one port to the chamber is mounted a source of vacuum ulraviolet radiatfon. Through another port (or palr or ports) we may direct the beam of an infrared spectrometer which measures the infrared absorptions in the sample on the cold finger in the range $2.5 \mathrm{\mu}-25 \mu\left(4000 \mathrm{~cm}^{-1}\right.$ to $\left.400 \mathrm{~cm}^{-1}\right)$. Th1s is the "finger-print" region for identifying molecules by their stretching, bending and rocking modes of oscillation in a solid. Other measurements are made of pressure, chemfluminescence, mass spectra, and visible absorption. Further descriptive detalls of the equipment as it existed several years ago may be found elsewhere [12].

TABLE 4. Comparison between Laboratory and Interstellar Conditions

Inttial

composition

thickress

temperature

Gas: pressure of condensible species

U1traviolet flux

$\lambda<2000$ A

Time scales

$$
\begin{aligned}
& \text { Diffuse clouds }{ }^{a} \text { ) } \\
& \text { Dense clouds }
\end{aligned}
$$

a) $\mathrm{n}_{\mathrm{H}}$

$100 \mathrm{~cm}^{-3}$

b) $\mathrm{n}_{\mathrm{H}} 1000 \mathrm{~cm}^{-3}$
$1 \mathrm{hr}$.

$1 \mathrm{hr}$.
Laboratory

$\mathrm{CO}, \mathrm{H}_{2} \mathrm{O}, \mathrm{NH}_{3}, \mathrm{CH}_{4} \cdots$

Interstellar

All condensible

species

$0.1 \mathrm{\mu m}$ to $10 \mu \mathrm{m}=0.1 \mu \mathrm{m}$

$>10 \mathrm{~K}$

$>10 \mathrm{~K}$

$10^{-7}$ mbar

$10^{15} \mathrm{~cm}^{-2} \mathrm{~s}^{-1}$

${ }^{3 n}\left[\right.$ H] $\times 10^{-20}$ mbar

$10^{8} \mathrm{~cm}^{-2} \mathrm{~s}^{-1}$

$10^{3}$ yrs.

$\sim 10^{4}-10^{6}$ yrs. 


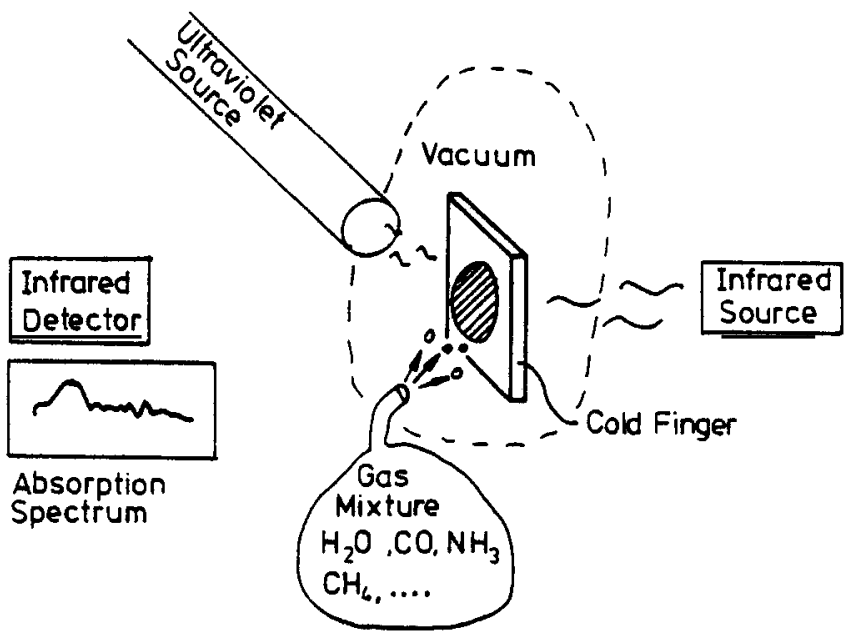

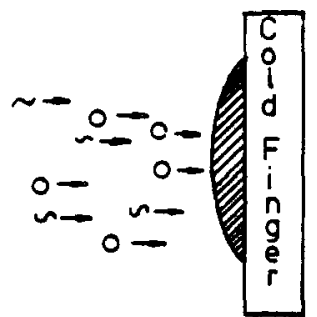

Laboratory

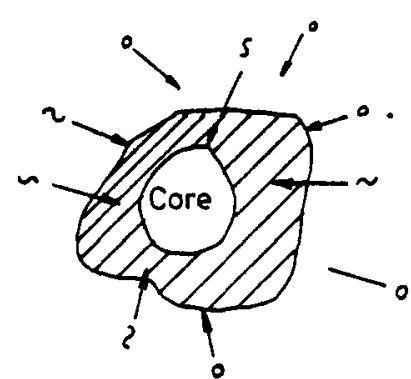

Interstellar

F18. 4. Schematic of the laboratory analog method for studying interstellar graln evolution. Molecules are deposited as a solld on a cold finger in a vacuum chamber and lrradiated by ultraviolet photons. The infrared absorption spectrum shows the appearance and disappearance of various molecules and radicals. The cold finger may be an aluminfum $(\sim 3 \mathrm{~cm})$ or a glass, sapph1re or LIF window.

A comparison between laboratory and interstellar conditions is seen in Table 4 . The most important, but necessary, difference is in the time scales for photoprocessing. One hour in the laboratory is equivalent to one thousand years in the diffuse cloud medium and as much as or greater than ten thousand to one million years in the depths of dense molecular clouds.

Types of Experiments. The basic mode of operation consists of deposition of mixtures of simple volatile molecules- $\mathrm{CH}_{4}, \mathrm{CO}, \mathrm{CO}_{2}, \mathrm{O}_{2}, \mathrm{~N}_{2}, \mathrm{NH}_{3}, \mathrm{H}_{2} \mathrm{O}-$ and simultaneous 1 rradiation as they freeze on the cold finger. Sometimes irradiation is continued after deposition is stopped. We simulate in this way the accretion and photoprocessing of grains in molecular clouds. The principal laboratory sequences and operations are: 1 ) Mensurement of the Infrared absorption spectra of unirradiated pure samples and intetures at $10^{\circ} \mathrm{K}$. Infrared studies also made of warmed and recooled samples. 2) Measurement of the infrared spectra following 1rradiation to detect presence of radicals and new molecules. 3) Measurement of the Infrared spectra of 1rradiated material following warmup to detect disappearance of frozen radicals and formation of new molecules. 4) Measurement of the visible absorption spectra of irradiated and wamed up samples. 5) Simultaneous measurement of chemfluminescence (vistble) and vapor pressure during warmup of 1rradlated samples. 6) Production of an explosion in the warmup period by thermally insulating the sample from the cold finger. 7) Taking infrared spectra and mass spectra of complex non-volatile residues remaining after warmup to room temperature. 8) Determining the visible absorption spectra of nonvolatile residues.

Some Experimental Results. The basic justification for the experiments which show how Interstellar grains evolve towards a chemical composition of preblological interest must come from comparison with astronomical observations. This has been discussed elsewhere 
[13]. I shall Ilm1t the discussion here to those aspects which bear more directly on the production of molecules of possible preblotic interest. In Figure 5 two sequences of irradiation of mixtures are shown by their infrared absorption spectra. In both sequences, some of the new species of molecules and radicals which appear followlng ultraviolet photolysis are labeled. In the upper sequence, an affect of particular note 1s that, following warmup, there appears a great change in the character of the spectrum (here shown only in the range $<1800 \mathrm{~cm}^{-1}$ but similar changes occur at higher frequencles). The features which appear after evaporation of the volatile components are much broader and are on their way to appearing as they do in the nonvolatile residue following total warmup (see Figure 6). In the lower pair of comparison spectra see the growth of the formaldehyde $\left(\mathrm{H}_{2} \mathrm{CO}\right.$ ) feature at around $6 \mathrm{~mm}$ and compare $1 \mathrm{t}$ with the $\mathrm{H}_{2} \mathrm{O}$ and $\mathrm{NH}_{3}$ features. It appears that $\mathrm{H}_{2} \mathrm{CO}$ is produced abundantly in situ within the grain analog material. The formaldehyde molecule is not terribly abundant in space, but so far no mechanism for its formation in the gas phase has proven adequate to account for what is observed consistent with its depletion by accretion on grains [14]. Consequently, its coplous production within the grains and a mechanism for molecule ejection is required. The latter is shown next.
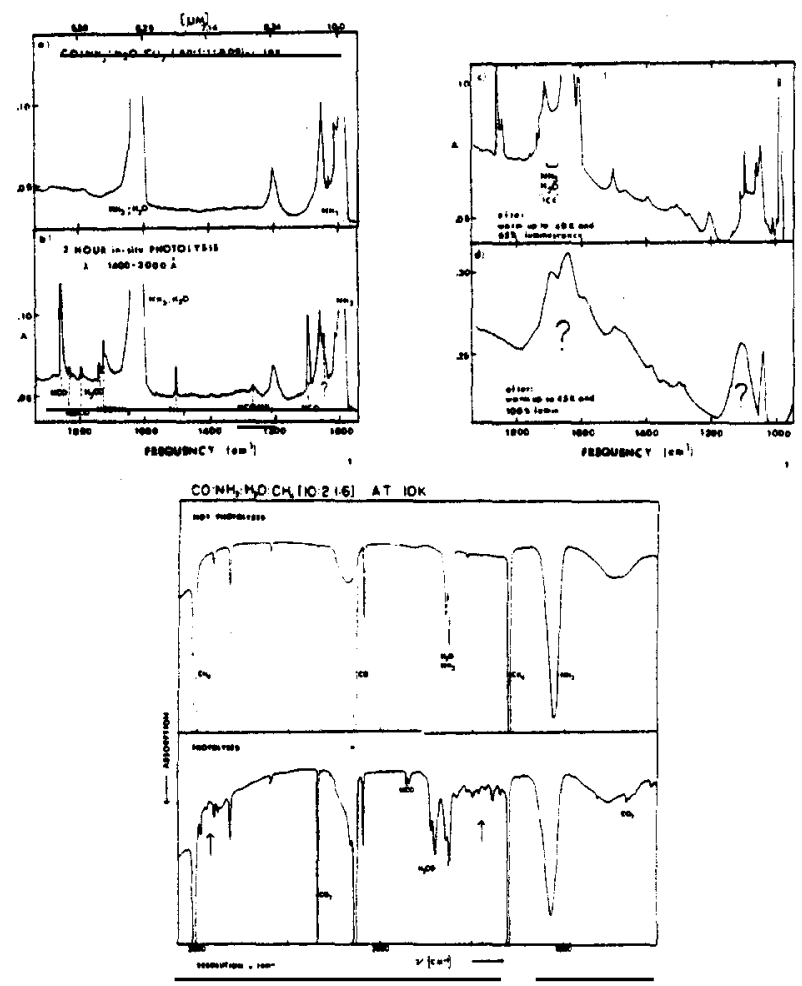

F1g. 5 Infrared absorption spectra of two sample analog grain mantles. Left side of upper sequence and the two lower spectra show first the features in unirradiated samples and then the spectra of the 1rradiated samples showing the appearance of new molecules and radicals produced by photoprocessing. Upper right hand pair of spectra clearly indicate the presence of complex molecules (unidentified) which appear as the more volatile specles are evaporated away by warming up.

We note that the frozen free radicals, exemplified here by the formyl radical HCo, appear Imediately in the infrared absorption spectrs of 1 rradiated samples. A special case of energy release by heating is the production of explosive reactions. Such reactions were predicted for 1nterstellar grains [15] which would explain not only the source of complex molecules in the interstellar gas but their ability to maintaln them against loss by depletion on the grains [16]. The experiments and application are being intensively pursued [17]. It has turned out that when the samples are warmed after photolysis they produce not only gradually varying luminescence and pressure but also spikes, indiceting violent chain reactions. The jor explosion always occurs at about $27^{\circ} \mathrm{K}$ wen the material is $11 t e r a l l y$ blasted off the cold finger. The critical fractional density of radicals required to produce explosions is estimated to be $\sim 10^{-2}$ and this occurs when the relative flux of photons to accreting molecules is about 1:10. This implies an efficiency for free radical production (net number of radicals/number of 1ncident photons) of about $10 \%$ which 16 approximately ten times higher than the conservative pre-experimental estimates [16]. 


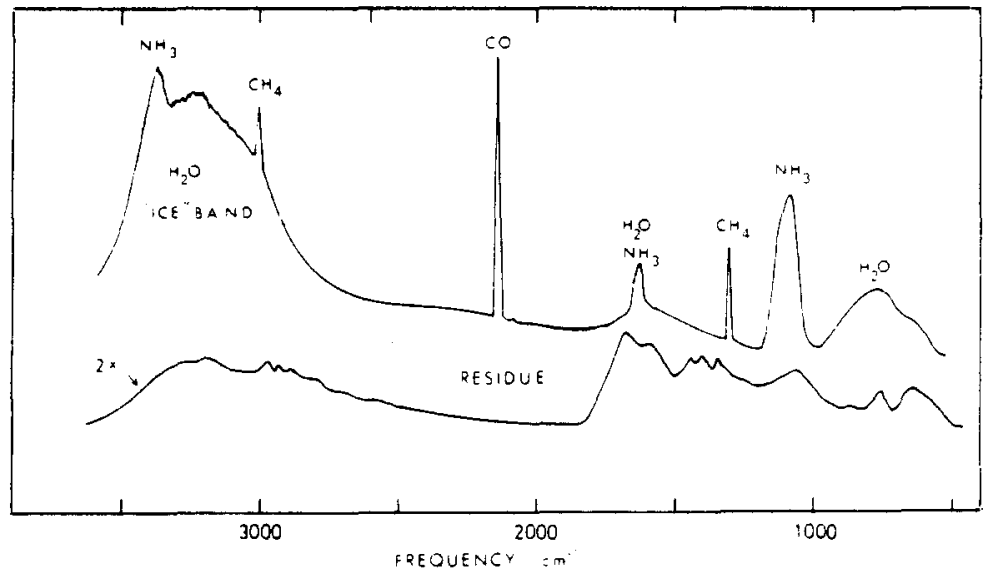

F1g. 6. Comparison of infrared absorption spectra of "yellow stuff" residue with $10 \mathrm{~K}$ mixture containing the same amount of oxygen, carbon and nitrogen in molecular form as in the initlal (pre-1rradiated) residue material. Note the complete absence of an $\mathrm{H}_{2} \mathrm{O}$ ice band at $3.08 \mu \mathrm{m}$ in the residue spectrum.

Subsequent to heating of 1rradiated samples, there always remains on the cold finger a yellow (near ultraviolet absorbing) nonvolatile residue. This residue remains indefinitely at room temperature and high vacuum $\left(10^{-6}\right.$ mbar). It is estimated that about $10 \%$ (2\% to $20 \%$ ) of the originally deposited volatile mpterials is converted into this yellow stuff in the interstellar equfvalent time of $\sim 10^{\prime}$ years (an astronomically reasonable time). The infrared absorption spectra of a varlety of materlals produced from complex mixtures are characterized by the general features shown in Figure 6. The very broad band peaking at about $3200 \mathrm{~cm}^{-1}$ is typlcal of carboxylic acld groups and some of the features around 1600$1700 \mathrm{~cm}^{-1}$ (see also Figure 5) are identiflable with amino groups. These types of spectra are also found in complex molecular mixtures created by entirely different means and presumed to be of preblological interest. See Flgure 7 for a typical example [18]. The very broad "3 $\mu \mathrm{m}$ " absorption $1 \mathrm{~s}$ not to be confused with the $\mathrm{H}_{2} \mathrm{O}$ ice band. Some of the smaller features around $3.4 \mu \mathrm{m}$ have been seen in space [19] and these are an indication that what we make in the laboratory is representative of what is made naturally in space and does not require Invoking the presence of bacterla or v1ruses [20].

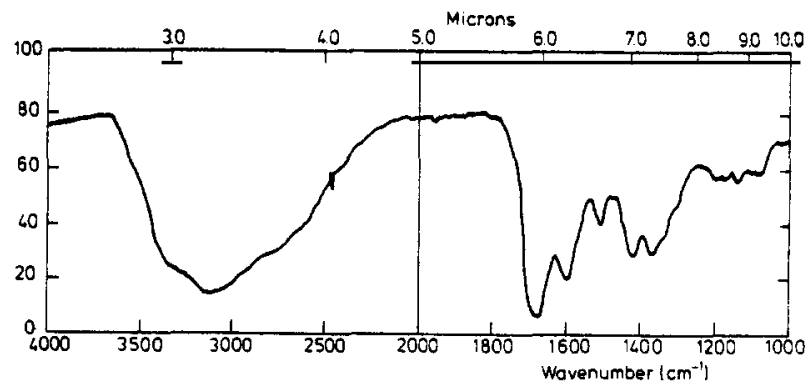

F1g. 7. Infrared absorption spectrum of an HCN-oligomerization product after Sephadex G-15 fractionation and HPLC separation. Indentified as 8-carbomoyl adenine (Figure courtesy of A.W. Schwartz and A.B. Voet). Th1s figure should be turned upside down to compare with Figure 6. 
One of our early resldues was found to have a molecular weight of 514 and its break-up components indicated 1 ts organic structure.

Insofar as the detalls of the chemical structure of our residues are concerned, a beginning has been made with some samples, one of which was examined by high resolution mass spectroscopy [20] and found to contain pieces, which appeared at about $400-500^{\circ} \mathrm{K}$, Identifiable as $\mathrm{C}_{4} \mathrm{H}_{6} \mathrm{~N}_{2}, \mathrm{CO}_{2}$ and trace urea $\left(\left(\mathrm{NH}_{2}\right)_{2} \mathrm{CO}\right)$. A tentative structure, as part of a polymer which contains amino pyroline rings, is given by

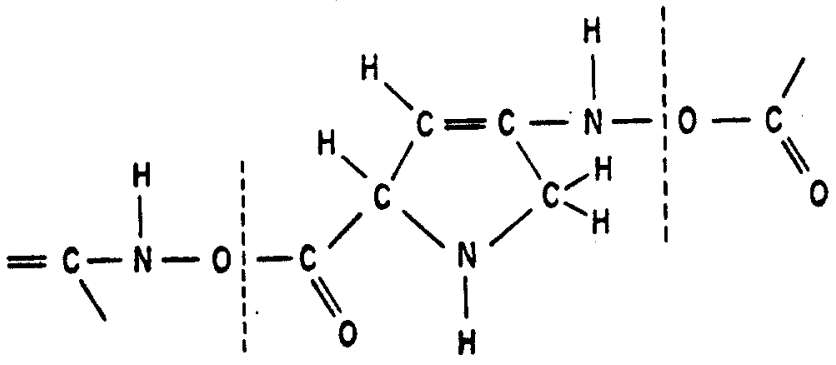

It 18 Interesting to speculate on this basis that we have a natural way of making porphyrins or porphyrin-ilke molecules in space as had been conjectured to exist some time ago [22]. An invest1gation 18 about to beg1n, with Bupport from the National Aeronautics and Space Agency, In which trace quant1t1es $(\sim 1 \%)$ of Fe will be deposited along with the $0, C, N$ bearing molecules. I anticipate the possibility that this will produce sone new and exciting results along these lines. The complex residues produced so far have all been water and methanol soluble.

EVOLUTION OF DUST.

Following the history of a typical interstellar grain we expect that it should have passed in and out of molecular clouds many times during its lifetime. It is only in the molecular clouds that it can accrete molecules. In the more tenuous region of space it is subjected to such a high ultraviolet flux relative to the collision rates of atoms that it can only survive if it is made of rather tough nonvolatile materials.

Chemical Composition of Dust in Diffuse Clouds. All attempts at observing the $\mathrm{H}_{2} \mathrm{O}$ ice band In diffuse clouds have proven negative. This is consistent with these dust gralns having had most of their volatiles, Including $\mathrm{H}_{2} \mathrm{O}$, sputtered away in the process of thelr ejection out of the molecular cloud phase. Since the absorption strength of the organic refractory stuff is weak, this in combination with the extreme broadness of its $3 \mu$ m "feature" make it rather difficult to detect. However, smaller absorption features appear to have been detected [19]. We conclude that the negative evidence for solid $\mathrm{H}_{2} \mathrm{O}$ in diffuse clouds may actually be providing us with positive evidence for the organic refractory materials, particularly if we add in the visible diffuse bands and the infrared bands to strengthen the argument.

Chemical Composition of Dust in Molecular Clouds. Although solld $\mathrm{H}_{2} \mathrm{O}$ is seen in the absorption of molecular cloud grains, it has been difficult in the past to estimate its abundance because of lack of data on its index of refraction in mictures. Leiden Astrophysics Laboratory data at $3 \mu \mathrm{m}$ of $\mathrm{H}_{2} \mathrm{O}$ containing mixtures has been used [23] to compare with the astronomlcal observation of the Becklln-Neugebauer (B.N.) protostellar object. See Table 5 for the distribution of the elements in the dust in a B.N. type molecular cloud. The result is that about 55\% of the outer mantle is in the form of amorphous $\mathrm{H}_{2} \mathrm{O}$ ice. This result is also consistent with calculations on grain mantle growth by surface reactions alone ignoring the photoprocessing [24]. Since the B.N. object is in a region of ongoing star formation, what we may be seeing are grains which are not in the molecular cloud steady state accretion-explosion phase, but rather grains which have grown in the last stages of final contraction and which are in a transition state between star formation and final ejection into the diffuse medium.

With or without this speculation, we can provide a substantial body of observational evidence which confirms the general ideas of photoprocessing of mixtures of oxygen, carbon and nitrogen bearing molecules in the varlous evolutionary phases of the interstellar dust, leading ultimately to a substantial grain component which is a complex organic material. 
TABLE 5. Elemental Composition of Gas and Dust in Protostellar Type Molecular Clouds Relative to Cosmic Abundances.

$\begin{array}{llllll}0 & \mathrm{C} & \mathrm{N} & \mathrm{Si} & \mathrm{Mg} & \mathrm{Fe}\end{array}$

Gas

\begin{tabular}{|c|c|c|c|c|c|c|c|}
\hline Atoms + Ions & - & - & - & - & - & - & (a) \\
\hline \multirow[t]{2}{*}{$\mathrm{CO}$} & 0.05 & 0.10 & - & - & - & - & \\
\hline & $(\sim 0.01)$ & $(\sim 0.03)$ & & & & & (b) \\
\hline Other & $<0.01$ & $<0.01$ & $<0.01$ & & & & (c) \\
\hline \multicolumn{8}{|l|}{ Molecules } \\
\hline \multicolumn{8}{|l|}{ Dust } \\
\hline Core + bare & 0.09 & 0.27 & & $\sim 1.0$ & $\sim 1.0$ & $\sim 1.0$ & \\
\hline Mantle & & & & & & & (d) \\
\hline Sol1d $\mathrm{H}_{2} \mathrm{O}$ & 0.22 & & & & & & \\
\hline OR & 0.11 & 0.42 & 0.22 & & & & \\
\hline Other & 0.05 & 0.11 & 0.26 & & & & \\
\hline Total Gas & 0.06 & 0.11 & 0.01 & & & & \\
\hline Total Solld & 0.47 & 0.80 & 0.48 & & & & \\
\hline Unaccounted & 0.47 & 0.09 & 0.51 & & & & \\
\hline Avpallable for & 0.53 & 0.20 & 0.52 & & & & \\
\hline
\end{tabular}

accretion

a) Not counting possible significant carbon fons as in Phillips et al. 1980, Astrophys. J. $238, \mathrm{~L} 107$.

b) If depletion of gas $C O$ in dense cores of molecular clouds (Rowan-Robinson, 1979, Astrophys. J. 234, 111).

c) Actually observed (see text).

d) Assumes extra grain mantle of $0.03 \mu \mathrm{m}$ and the ice absorption for B.N. (see text).

Paraphrasing a nursery rhyme: What are 11ttle grains made of? Organfcs and 1ce and everything nice, that's what 11ttle grains are made of. Typical interstellar grains might be represented as in Figure 8 .
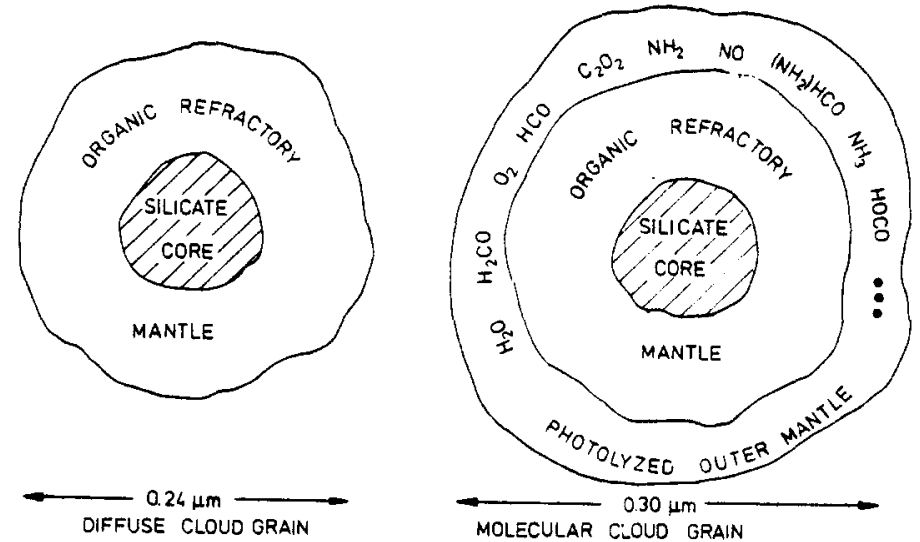

F1g. 8. Chemical models of interstellar grains in diffuse and molecular clouds. The molecular cloud mantle size shown here corresponds approximatediy to those in the BN type clouds. In protostellar clouds the outer mantle could be thicker. 
INTERSTELLAR DUST ON THE EARTH.

How Much Organic Material is in a Cloud? As the Earth revolves around the sun, and the solar system as a whole revolves around the center of the Milky Way, we are continually passing through the gas and dust in space. The solar system at the current epoch is in the midst of the most tenuous kind of gas so that the interstellar dust can barely make its presence felt or observed and then only by measurements made outside of the earth's orbital plane. However at quite a few times in the past, the solar system was immersed in dense molecular clouds from whlch it could have gathered large quantities of dust and gas. Although we know the gas to contain many interesting organic molecules, including $\mathrm{H}_{2} \mathrm{CO}$ and HCN, which can play a role in prebiotic chemistry, we also realize that the total fraction of available $C, O$, and $N$ seen in these molecules is quite small compared to what we infer to be in the dust. Consequently, although we may use the gas phase molecules as an indicator, we will consider that the bulk of molecules are bound in the dust.

In the steady state regime in molecular clouds, the accretion-explosion balance cycles roughly $75 \%$ of the cosmically abundant atoms and molecules containing 0 , $C$ and $N$ as volatiles between the gas and dust. The remaining $\sim 25 \%$ are in the organic refractory yellow stuff of the inner grain mantle. Of the $75 \%$ labile material, approximately $50 \%$ is at any moment on the grain 80 that $\sim 65 \%$ of the total are in the dust [17]. From th1s, we conservatively estimate that $0.1 \%$ of all the mass of our entire Milky Way 18 in the dust. For example, in a molecular cloud of l parsec radius ( $\sim 3$ light years) and a hydrogen density $n_{H}=10^{4} \mathrm{~cm}^{-3}$, the complex organic molecules alone of which about $1 / 2$ may be the very complex yellow stuff, account for a mass equal to that of our sun.

Accretion of Interstellar Matter by the Primitive Earth. There are many ways by which matter from space has been, and still 1s, deposited on the Earth. For example, when the Earth was first formed, there were enormous quantities of debris still remaining in the solar system which bombarded the Earth's surface [25]. Furthermore, since the solar system may be presumed to have formed and remalned for several million years within a moleculr cloud complex it would have continued to accrete large quantities of interstellar matter during this time. However, the current thinking about the state of the Earth's surface at such an early epoch makes it highly unlikely that even abundant deposits of preblotic materlal could have elther survived or found sultable environment. On the other hand, since there now appears to be evidence for life having already been present on the Earth some 3.8 billion years ago we must limit ourselves to the questions of when and how preblotic matter could have been deposited on the terrestrial surface during this first 700 million years and actually perhaps in an even narrower time frame between 300 and 700 million years. I shall consider here only the accretion during passage of the solar system through dense clouds. Clouds of comets have been considered elsewhere [26].

It turns out that at the distance of the sun from the galactic center, the solar system is rotating about the galactic center at about twice the angular speed of the spiral pattern. Therefore, since the pattern consists of two main spiral arms and since the galactic rotation period of the solar system is about 200 million years [27], the sun and Earth pass through a relatively high concentraton of dust and gas at the inner edges of spiral arms (see Figure 1) every 110 million years. This is shown schemaricaliy in figure 9. We have thus passed through a spiral arm some 40 times since the formation of the solar system. It can be estimated [28] that at the present epoch the collision probability of the earth with clouds of hydrogen density $\mathrm{n}_{\mathrm{H}} \sim 2 \times 10^{3} \mathrm{~cm}^{-3}$ is about 0.25 for each passage through a spiral arm. As a rough approximation we may assume that $4 \times 10^{9}$ years ago the amount of interstellar matter was about twice 1ts present value, so that during the first several hundred mfllion years the probability for the cloud encounters was about twice as large as now. The number of clouds with density $\mathrm{n}_{\mathrm{H}} \sim 10^{4} \mathrm{~cm}^{-3} 18$ somewhet less certain than that for the lower densities because of observational selection effects. However, rough approximation to the fall-off in cloud number density is that it decreases inversely as matter density. We conclude then that during the first 700 million years the probability was of the order of unity that the Earth passed through one very dense $\mathrm{cloud}\left(\mathrm{n}_{\mathrm{g}}>10^{4} \mathrm{~cm}-3\right)$ and that the passage through clouds of density $\mathrm{n}_{\mathrm{H}}>10^{3} \mathrm{~cm}^{-3}$ probably occurred as many as 3-4 times. We 1gnore the clouds of lower density because they probably deposit material at too low a rate to be important. Perhaps this should be examined further because these clouds are far more abundant.

The rate of input of complex preblotic type dust molecules during cloud pasage is in the range of $10^{3}$ to $10^{4}$ metric tons ( 1 metric ton $=10^{6} \mathrm{~g}$ ) per yeer.

Since the passage time through a typical cloud is $10^{5}-10^{6}$ years, the total deposition 18 between $10^{8}$ and $10^{10}$ ton which is $10^{-14}$ to $10^{-12}$ of the Earth's total mass. This $1 \mathrm{~s}$ far greater than the current blomass of the Earth. 


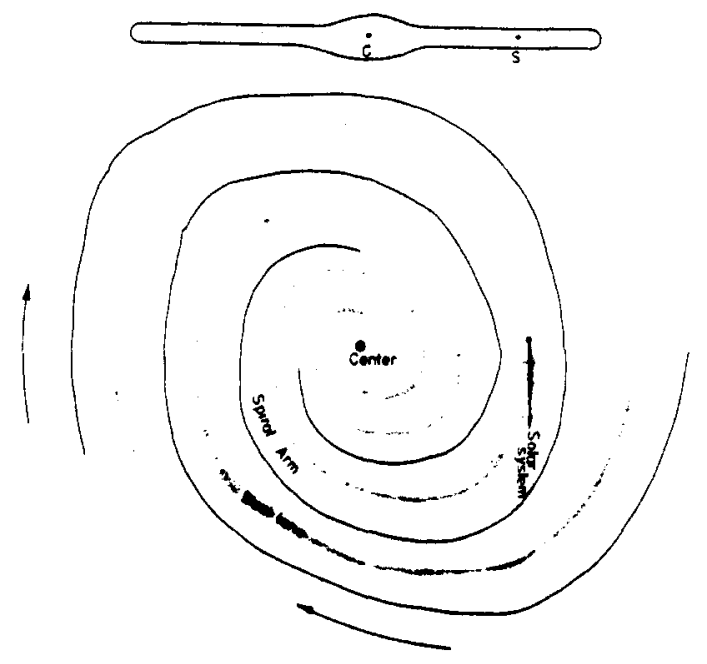

F1g. 9. Schematic diagram of a spiral galaxy seen edge-on and face-on showing the concentration of dust. The position and relative veloctity of the solar system with respect to the spiral pattern is 1llustrated at a time when ft was passing through a region where the dust clouds are concentrated.

We may probably assume no modification of the chemical composition of the dust as it Impinges on the Earth Any existing atmosphere would act like a cushion slowing down these submicron particles without significantly heating them as occurs for much larger meteors and even the interplanetary dust [29]. However, al though the nonvolatile component would be unaffected, the more volatile constituents in the outer mantle of the dust would have partially, if not totally, evaporated because of heating by the sun.

Interstellar Dust in Comets. It is currentiy belfeved that comets are a good representative sample of the 1nterstellar matter out of which they form. As such, their chemical composition is basicaliy that of the interstellar dust at the final stage in the prestellar nebula.

This composition is 11lustrated in Table 6 where it is assumed that all the atoms and molecules in the gas have accreted on the dust. Comet collisions with the earth have undoubtedly contributed substantial quantities of organic material in the past and Indeed, many have contributed as much or more than that by the direct accretion of the interstellar dust. However the explosive reaction which occurs on Impact of a comet likely would lead to sufficient heating to pyrolize the complex molecules. Nevertheless a relatively high local concentration of organfc material should result and perhaps be conduclve to rap1d continuing chemfcal evolution.

TABLE 6. Suggested Chemical, Mass and Volume Distribution of the Principal Condensable Atomic Consituents in a Comet.

$\begin{array}{lcll}\begin{array}{l}\text { Component } \\ \text { Fraction }\end{array} & \text { Mass } & \begin{array}{l}\text { Density } \\ \text { Fraction }\end{array} & \text { Volume } \\ \text { S1licates } & & 3.5 & 0.086 \\ \text { Carbon } & 0.21 & 2.5 & 0.034 \\ \text { Very complex OR } & 0.06 & \sim 1.3 & 0.21 \\ \mathrm{H}_{2} \mathrm{O} & 0.19 & 1 & 0.27 \\ \mathrm{CO} & 0.19 & 1.05 & 0.13 \\ \text { Other molecules + radicals } & 0.10 & 1.3 & 0.27\end{array}$

$\left(\mathrm{CO}_{2}, \mathrm{~N}_{2}, \mathrm{HCN}, \mathrm{H}_{2} \mathrm{CO}, \mathrm{HCO}, \ldots\right)$ 
CONCLUSION

The chemical evolution of Interstellar matter via gas phase and solid reactions leads to an enormous reservolr of organic molecules in the space between the stars. The impressive sampling of organic molecules seen by radioastronomical methods in the gas is but a 8 mall part of the total of which, by mass and complexity, the major portion floats about in small frozen submicron sized particles of interstellar dust. The accretion of substantial masses of Interstellar dust from space by a planet like the Earth occurs with high probability in the early stages of crustal development. It is suggested that the high degree of complexity of the dust organics may have been adequate to provide the chemical templates leading to the origin of life at the first opportune time in the Earth's formation, perhaps previous to very substantial complex molecule formation in a primitive sterrestrial atmosphere. The question of whether the ultimate dust composition is so ordered as to give such a head start can only be established by further intensive laboratory studies in solid phase photochemical evolution of interstellar dust particles.

\section{ACKNOWLEDGEMENTS}

This work has been supported in part by grants from the Netherlands Organization for Pure Research ( $2 \mathrm{WO}$ ). The contributions by all my colleagues in the department of Laboratory Astrophysics at Lelden Univeraity and by Professor De Jong in the department of Pharmocology are gratefully acknowledged.

\section{REFERENCES}

1. L.E. Snyder, D. Buhl, B. Zuckerman and S. Palmer, Phys, Rev. Letters 22, 679 (1969).

2 A.P.C. Mann and D.A. W1ll1ams, Nature 283, 721 (1980).

3. J.M. Greenberg, Astron. Astrophys. 12, 240 (1971).

4. S.S. Hong and J.M. Greenberg, Astron. Astrophys. 88, 194 (1980).

5. H.C. de Hulst, Rech. Astr. Obs. Utrecht 11 , part $\frac{8}{2}$ (1949).

6. H.J. Hablng, Bol. Ast. Inst. Ned. $19,42 I$ (1968).

7. G.C. P1mentel, in Formation and Trapping of Free Radicals, eds. A.M. Bass and P. Brolda, (Academic Press, N.Y.) chapter 4, p. 69, 1960

8. E.R. Danielson, N.J. Woolf and E.J. Gaustad, Astrophys. J. 141, 116 (1965).

9. See 1 .

10. J.M. Greenberg, A.J. Yencha, J.W. Corbett and H.L. Frisch, Mem. Soc. Roy. Sclences Liege, 6e serie, Tome III, 425-463, 1972.

B. Khare and C. Sagan, In Molecules in the Galactic Environment, eds. M.A. Gordon and L.E. Snyder (W1ley and Sons, N.Y.), 1973.

11. S.L. Miller, Science, 117,528 (1953).

12. W. Hagen, L.J. Allamandola and J.M. Greenberg, Astrophys. \& Sp. Sc1. 65, 215 (1979).

13. J.M. Greenberg, in Submill1metre Wave Astronomy, eds. J.P. Ph1111ps and J.E. Beckman, Cambridge Unfversity Press, 1982 , In press.

14. T. de Jong, A. Dalgarno and W. Boland, Astron. Astrophys. 91, 68 (1980).

15. J.M. Greenberg, in Molecules in the Galact1c Environment, eds. M.A. Gordon and L.E. Snyder, J. Wiley and Sons, N.Y., 1973, p. 94 .

16. J.M. Greenberg, In Stars and Star Systems, ed. B.E. Westerlund, Re1del, Dordrecht, 1979 , p. 177.

17. L. d'Hendecourt, L.J. Allamandola, F. Baas and J.M. Greenberg, Astron. Astrophys. (1982) in press.

18. A.W. Schwartz and A.B. Voet, Private communication

19. D.A. Allen and D.T. W1ckramasinghe, Nature 94,539 (1981). C.E.P.M. van de Bult, L.J. Allamandola and J.M. Greenberg, A laboratory analysis in progress.

20. F. Hoyle, M.C. Wickramasinghe, S. Al-Mufit, A.H. Olavesen and D.T.Wickramasinghe, Astrophys. Sp. Sci. (1982) in press.

21. Work in collaboration with Professor H.J. de Jong, Leiden.

22. F.M. Johnson, in Interstellar Grains, eds. J.M. Greenberg and T.P. Roark, NASA SP140,1967, p. 229.

23. J.M. Greenberg, in Comets, ed. L. Wilkening (Univ. of Arizona Press), 1982, p. 131. W. Hagen, A.G.G.M. Tielens and J.M. Greenberg, Astron. Astrophys. (1982) accepted for publication.

24. A.G.G.M. THelens and W. Hagen, Astron. Astrophys. (1982) submitted.

25. D.G. Ashworth, in Cosmic Dust, ed. J.A.M. McDonne11, (J. Wiley \& Sons), 1978, p. 427.

26. C. Ponnamperuma, ed. Comets and the 0rigin of Life, (Reidel, Dordrecht), 1981.

27. B.J. Bok, Scientific American, March, 1981.

28. R.J. Talbot, Jr. and M.J. Newman, Astrophys. J. Supplement 34, 295 (1977).

29. D. Brownlee, in Cosmic Dust, ed. J.A.M. McDonnel1, J. Wiley, N.Y., 1978, p. 295. 